\title{
Geometrically Nonlinear Theory of Composite Beams with Deformable Cross Sections
}

\author{
Rafael Palacios* and Carlos E. S. Cesnik \\ University of Michigan, Ann Arbor, Michigan 48109-2140
}

DOI: $\underline{10.2514 / 1.31620}$

\begin{abstract}
A one-dimensional theory of slender structures with heterogeneous anisotropic material distribution is presented. It expands Cosserat's description of beam kinematics by allowing deformation of the beam cross sections. For that purpose, a Ritz approximation is introduced on the cross-sectional displacement field, which defines additional elastic degrees of freedom (finite-section modes) in the one-dimensional model. This results in an extended set of beam dynamic equations that includes direct measures of both the large global displacement and rotations of a reference line, and the small local deformations of the cross sections. Two situations are studied in which this approach provides a simpler alternative to shell models with comparable fidelity. First, we look at the detailed structural response of composite beams with distributed loads. In particular, the case of a composite box beam with embedded piezoelectric actuators is considered. Second, this methodology is applied to study the low-frequency response characterization of composite beams. Numerical results in both cases show that a reduced set of finite-section modes allows a full description of the actual three-dimensional displacement field using a strictly one-dimensional formulation.
\end{abstract}

\section{Nomenclature}

$A=$ area of the cross section

$\mathbf{a}_{\mathbf{i}}=$ unit vectors of the reference coordinate system

$\mathbf{B}_{\mathbf{i}}=$ unit vectors of the local coordinate system in the deformed reference line

$\mathbf{b}_{\mathbf{i}}=$ unit vectors of the local coordinate system in the undeformed reference line

$\mathbf{C}^{\text {sr }}=$ rotation tensor from coordinate systems $r$ to $s$

c $=$ tensor of material elastic constants

$\mathbf{e}_{\mathbf{i}}=$ unit vector along the $i$-direction

$\mathbf{F}=$ vector of cross-sectional internal forces

$f_{s_{0}}=$ column matrix of applied conjugated forces of $q$

$f_{s_{1}}=$ column matrix of applied conjugated forces of $q^{\prime}$

$\mathbf{f}_{\mathbf{0}}=$ vector of zero-order applied forces per unit length

$\mathbf{f}_{1}=$ vector of first-order applied forces per unit length

$g \quad=$ damping coefficients

$\mathbf{H}=$ vector of sectional inertial angular momenta

$h=$ characteristic dimension of the cross section

$\mathcal{K}=$ kinetic energy per unit length

$\mathbf{K}=$ curvature vector in the deformed reference line

$\mathbf{k}=$ curvature vector of the undeformed reference line

$l=$ beam length

$\mathbf{M}=$ vector of sectional internal moments

$M=$ sectional mass matrix

$\mathbf{m}_{\mathbf{0}}=$ vector of zero-order applied moment per unit length

$\mathbf{m}_{\mathbf{1}}=$ vector of first-order applied moment per unit length

$\mathbf{P}=$ vector of sectional inertial linear momenta

$Q_{s_{0}}=$ column matrix of conjugated forces of $q$

$Q_{s_{1}}=$ column matrix of conjugated forces of $q^{\prime}$

Presented as Paper 2275 at the 48th AIAA/ASME/ASCE/AHS/ASC Structures, Structural Dynamics, and Materials Conference, Honolulu, Hawaii, 23-26 April 2007; received 15 April 2007; revision received 10 September 2007; accepted for publication 2 October 2007. Copyright $\odot 2007$ by Rafael Palacios and Carlos E. S. Cesnik. Published by the American Institute of Aeronautics and Astronautics, Inc., with permission. Copies of this paper may be made for personal or internal use, on condition that the copier pay the $\$ 10.00$ per-copy fee to the Copyright Clearance Center, Inc., 222 Rosewood Drive, Danvers, MA 01923; include the code 0001-1452/08 $\$ 10.00$ in correspondence with the CCC.

*Postdoctoral Research Fellow, currently Lecturer, Imperial College London, Department of Aeronautics, London SW7 2AZ, United Kingdom; rpalacio@imperial.ac.uk. Member AIAA.

†Associate Professor, Department of Aerospace Engineering; cesnik@ umich.edu. Associate Fellow AIAA.
$Q_{t}=$ column matrix of conjugated momentum of $\dot{q}$

$q=$ column matrix with amplitudes of finite-section modes

$\mathbf{R}=$ position vector at the deformed reference line

$\mathbf{r}=$ position vector at the undeformed reference line

$S=$ sectional stiffness matrix

$t=$ time

$\mathcal{U}=$ strain energy per unit length

$\mathbf{u}=$ displacement vector of the origin of reference coordinate system

$\mathbf{V}=$ inertial velocity vector at the deformed reference line

$\mathbf{v}=$ inertial velocity vector of origin of reference coordinate system

$\mathbf{w}=$ warping displacement vector

$\mathbf{X}=$ position vector of the deformed material point

$\mathbf{x}=$ position vector of the undeformed material point

$x=$ curvilinear coordinate along the undeformed reference line

$x_{\alpha}=$ cross-sectional coordinates in the reference configuration

$\Gamma=$ strain tensor in the three-dimensional solid domain

$\gamma=$ vector of force strain measures at reference line

$\delta \quad=$ variational operator

$\overline{\delta \mathcal{A}}=$ applied action at time and spatial boundaries

$\overline{\delta \mathcal{W}}=$ virtual work per unit length of the external forces

$\overline{\delta \Phi}=$ column matrix of local virtual rotations of reference line

$\overline{\delta \varphi}=$ column matrix of virtual rotations of reference coordinate system

$\kappa=$ vector of moment strain measures at reference line

$\mu=$ vector of distributed applied force per unit volume

$\rho \quad=$ mass density

$\Psi=$ matrix of approximating functions for finite-section modes

$\boldsymbol{\Omega}=$ inertial angular velocity vector at the deformed reference line

$\omega=$ inertial angular velocity vector of the reference coordinate system $(a)$

$(\tilde{\bullet})=$ cross-product operator

(•) $=$ differentiation with respect to $t$

$(\bullet)^{\prime}=$ differentiation with respect to $x$

\section{Introduction}

A lthough beam models allow the easy modeling of structural elements with a dominant spatial dimension, their application is typically limited by additional kinematic constraints to the 
deformation. One typical assumption is that the deformation of the beam reference line defines the displacement field in the actual threedimensional slender solid by assuming, for instance, rigid cross sections (Cosserat's model). The resulting models are valid while the area of the cross section remains close to zero, but it is not that obvious to establish their limits when the cross-sectional size increases. The aim of this paper is to establish a theory of beams with finite-size cross sections that satisfies the following requirements: 1) it uses a one-dimensional representation of the structure along a reference line; 2) it allows for deformations of the cross section not necessarily related to the deformation of the reference line; and 3) it accounts for cross sections of arbitrary shape and material distribution for initially curved or twisted reference lines, and for arbitrarily large deformations of the reference line.

Basic concepts of beam theory were first developed for the modeling of solid homogeneous isotropic structures. There we find the classical linear beam theories used in strength of materials, that is, the Euler-Bernoulli or Timoshenko theories. They are displacement formulations; an ad hoc approximation of the cross-sectional displacement field yields the strain energy and, using energy principles, some beam stiffness relations can be defined, as well as the subsequent equations of motion. Those classical assumptions in the displacement field, however, no longer represent the behavior of anisotropic slender structures. The concept of warping, which was first developed for the Saint-Venant theory of torsion, is used to quantify this mismatch and, based on it, a number of composite beam theories have been formulated. With an interest in helicopter blade design, Volovoi et al. [1] and Jung et al. [2] have reviewed the available modeling methods for anisotropic beams.

Asymptotic expansions provide a useful mathematical tool to construct one-dimensional structural models from the threedimensional equations of elasticity without ad hoc assumptions in the displacement field. The slenderness of the structure provides a small parameter $(h / L$, with $h$ being the cross-sectional typical dimension and $L$ the characteristic wavelength of the deformation) to build an asymptotic approximation to the solution of the three-dimensional elastic problem. This is done without assumptions on the displacement field, which is approximated as part of the asymptotic solution process. The problem can be posed from either a weak [ $3-5]$ or a strong [ [ $\underline{6}-\underline{8}]$ form of the equations of elasticity and its solution, except for a few particular configurations, needs to be obtained numerically. In general, this problem can be seen as the estimation of how much the actual displacement field in the slender solid deviates from the rigid cross sections along a deformable reference line (which corresponds to the limit $h / L \rightarrow 0$ ). These local deformations are normally referred to as warping displacements of the beam cross sections. The first warping model was postulated by Saint-Venant to capture the local shear strains in the torsion of isotropic beams (see Love [9], paper 82). This defines, in general, a two-dimensional problem in the cross sections that also appears with transverse shear deformations, and for which different numerical solutions have been proposed $[10,11]$. For the case of anisotropic slender structures, a warping correction appears on the cross sections for each possible deformation of the reference line (its bending, extension, shear, and twist degrees of freedom) and more general solutions have been proposed for the warping field $[\underline{4}, \underline{12}, 13]$. They are based on different homogenization procedures of the local three-dimensional equilibrium equations per unit length of the slender solid. In [12], the warping field is obtained through minimization of the energy per unit length, but the cross-sectional and longitudinal problems are coupled in the solution, which complicates the actual implementation of the method. This is avoided in [13] through the identification of the beam motions as the kernel of the equations in the interior of the solid, which implies a variational definition of the onedimensional deformations of the prismatic beam without assumptions on the displacement field. Alternatively, an asymptotic solution is proposed in [4] by applying the variational-asymptotic method [3] to anisotropic beams. This results in the variationalasymptotic beam cross-sectional analysis, which was originally based on four classical beam elastic degrees of freedom (extension, twist, and bending in two directions). One big advantage of the variational description is that it provides a robust framework for more general formulations and thus the model in [4] was later expanded $[14,15]$ to account for a finite number of additional (higher-order) nonclassical one-dimensional elastic degrees of freedom. These nonclassical deformations include situations such as the camberbending deformation of thin strips [16] or the generalization of the Vlasov correction for open-cell composite beams of Volovoi and Hodges [17]. Cesnik et al. [14] proposed an eigenvalue problem on the cross-sectional displacement field and therefore nonclassical modes that are independent on the actual loading on the beam. To improve this, Palacios and Cesnik [15] substituted them with a Ritz approximation in which assumed shape functions on the warping field (finite-section modes) are used to represent cross-sectional deformations of interest for a given configuration. This results in a theory of beams with arbitrarily deformable cross sections, which will be fully developed in this work.

The proposed formulation is based on a spatial reduction of the three-dimensional equations of motion of the slender solid, which are reduced to describe the evolution of certain magnitudes (both the classical and nonclassical beam deformations) along a reference line. This reference line will be allowed to move in space and have arbitrarily large deformations. In that respect, the classical part of the deformation follows Cosserat's model, that is, the beam is represented by a deformable line curved in space with known elastic properties and the deformation of the curve is analyzed using the tools of differential geometry (see Love [9], paper 254). Using this approach, Reissner [18] determined the geometrically exact static equations of equilibrium, whereas a geometrically exact model for beam dynamics was later introduced by Simo [19]. Those are intrinsic formulations, but are explicitly solved in displacements/ rotations. Hodges [20] derived a mixed form of the beam dynamic equations, which allows a very simple numerical solution scheme [21]. This model will be expanded here to include also the deformation of the cross section through a set of finite-section modes. The resulting one-dimensional formulation explicitly captures both the large elastic deformations of the beam reference line as well as the small local deformations at the cross section. Typical situations in which finite-section modes may be desirable are the accurate estimation of the local deformation of composite beams under nonuniform distributed loads (for example, bimoments acting on a thin strip) and dynamic response of composite beams at low-tomoderate frequencies. In those situations, the conventional solution would be based on a shell model and this is avoided here by defining a set of finite-section modes. Both situations are exemplified with numerical tests in this paper. Applications under study (an on-going research) of this approach also include the structural models in aeroelasticity of rotorblades with either computational fluid dynamics (CFD) [22] or flexible-airfoil potential [23] aerodynamics.

\section{Theoretical Development}

Starting from the three-dimensional elastodynamic equations, the slenderness of the structure defines a small parameter in the equations. This identifies two scales in the analysis and splits the problem into a long-scale one along the longitudinal dimension and a small-scale one at each cross section. A previous work by the authors [15] has presented the general solution to the cross-sectional problem and this work will focus on details of the development of the onedimensional dynamic equations along the reference line. For completeness, some key results on the reduction process from the three-dimensional solid to the reference line are also included here.

\section{A. Kinematics}

Consider the two configuration states in the deformation of a slender solid of slowly varying cross section as shown in Fig. 1. They correspond to the undeformed (reference) and deformed (current) configurations. The deformation is described using a Lagrangian description, with lower- and upper-case symbols used for magnitudes at the undeformed and deformed state, respectively. The description is done from the viewpoint of an observer on a moving reference frame $(a)$. The motion of this frame is given with 


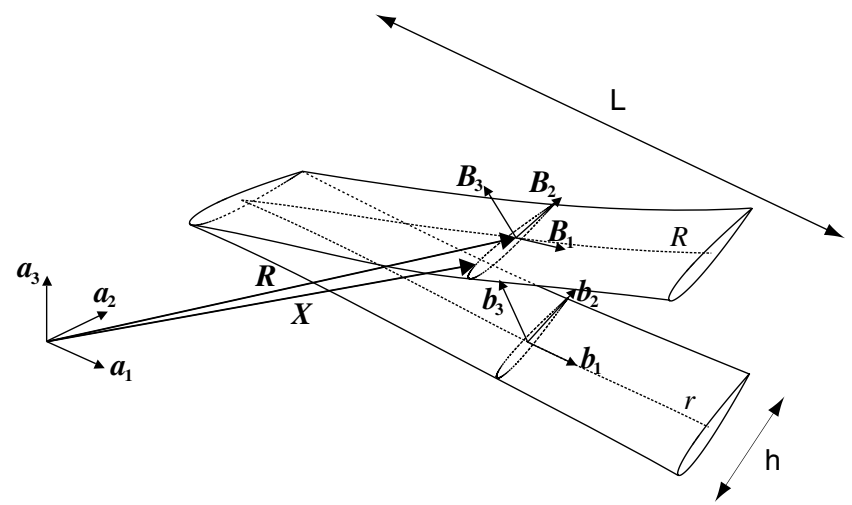

Fig. 1 Coordinate frames in the deformation of a slender solid.

respect to an inertial frame (a certain global frame, o) by the translational velocity of its origin, $\mathbf{v}$, and its rotational velocity, $\omega$, which, in general, are unknown.

A (curved) reference line, $r$, is defined along the longitudinal dimension of the undeformed configuration. Let $\mathbf{r}$ and $\mathbf{x}$ be the position vectors along the reference line and in the undeformed solid, respectively. Curvilinear coordinates can be defined, such as $x=x^{1}$ is the coordinate along $r$ and $x_{\alpha}=x^{\alpha}$ are the orthogonal coordinates in its normal planes. The tangent vectors at the reference line to the three curvilinear coordinates define the undeformed reference frame, $b$. The position vector $\mathbf{x}$ in the undeformed solid can then be written as

$$
\mathbf{x}\left(x, x_{2}, x_{3}\right)=\mathbf{r}(x)+x_{a} \mathbf{b}_{\alpha}(x)
$$

Let $\mathbf{X}$ be the position vector in the current configuration of a material point initially at $\mathbf{x}$. We define now the deformed reference line, $R$, by the averaged value of $\mathbf{X}$ on the cross sections of $r$ :

$$
\mathbf{R}(x)=\frac{1}{A(x)} \int_{A(x)} \mathbf{X} \mathrm{d} A
$$

where $A(x)$ is the cross-sectional area at $x$. In general, the covariant base vectors corresponding to the curvilinear coordinates $\mathbf{X}\left(x_{i}\right)$ will not define an orthogonal basis. To simplify the description we introduce an "intrinsic" orthogonal deformed reference frame, $B$. Let $\mathbf{Z}=\mathbf{X}-\mathbf{R}$ be the position vector in the deformed cross section. Frame $B$ is defined by enforcing that the average rotation around the three vectors $\mathbf{B}_{\mathbf{i}}$ is zero, that is,

$$
\begin{gathered}
\int_{A(x)}\left(x_{3} \mathrm{Z}_{2}-x_{2} \mathrm{Z}_{3}\right) \mathrm{d} A=0 \\
\int_{A(x)} x_{3} \mathrm{Z}_{1} \mathrm{~d} A=0, \quad \text { and } \quad \int_{A(x)} x_{2} \mathrm{Z}_{1} \mathrm{~d} A=0
\end{gathered}
$$

where $\mathbf{Z}=Z_{i} \mathbf{B}_{\mathbf{i}}$. Following Hodges [24], the rotation matrices between the global frame $(a)$ and the undeformed $(b)$ and deformed $(B)$ frames will be denoted as $C^{b a}(x, t)$ and $C^{B a}(x, t)$, respectively, and their spatial derivative along the reference line defines the curvature vectors of the undeformed $\left(\mathbf{k}=k_{b, i} \mathbf{b}_{\mathbf{i}}\right)$ and deformed $\left(\mathbf{K}=K_{B, i} \mathbf{B}_{\mathbf{i}}\right)$ reference lines, respectively. The position vector in the current configuration of the material point initially at $\mathbf{x}$ can be written, without loss of generality, as [15]

$$
\begin{aligned}
& \mathbf{X}\left(x, x_{2}, x_{3}\right)=\mathbf{R}(x)+x_{\alpha} \mathbf{B}_{\alpha}(x)+q_{n}(x) \Psi_{n, i}\left(x_{2}, x_{3}\right) \mathbf{B}_{\mathbf{i}}(x) \\
& \quad+w_{i}\left(x, x_{2}, x_{3}\right) \mathbf{B}_{\mathbf{i}}(x)
\end{aligned}
$$

where $\Psi_{n, i}$, with $n=1, \ldots, N$, is a set of approximating functions to the sectional deformation field to capture nonclassical deformations (which, in what follows, are referred to as finite-section modes); $q_{n}$ are the amplitudes of those finite-section modes; and $\mathbf{w}=w_{i} \mathbf{B}_{\mathbf{i}}$ is the residual warping displacement vector. Note that, if the finite-section modes are zero and the warping is the (prescribed) Saint-Venant torsion warping, Eqs. (3) and (4) correspond to the kinematic assumptions for the Timoshenko model of an isotropic beam with extension, bending, shear, and twist degrees of freedom. In the general case, for other values of these variables, several orthogonality constraints need to be imposed on them to give a unique description of the deformation. They are chosen as [15]

$$
\begin{aligned}
& \int_{A(x)} \bar{\Psi}^{T} w \mathrm{~d} A=0 \\
& \int_{A(x)} \Psi^{T} \Psi_{0} \mathrm{~d} A=0
\end{aligned}
$$

where

$$
\begin{aligned}
& \Psi_{0}=\left[\begin{array}{cccccc}
1 & 0 & 0 & -x_{2} & x_{3} & 0 \\
0 & 1 & 0 & 0 & 0 & -x_{3} \\
0 & 0 & 1 & 0 & 0 & x_{2}
\end{array}\right] \\
& \bar{\Psi}=\left[\begin{array}{lll}
\Psi_{0} & \mid & \Psi_{0}
\end{array}\right], \text { and } \quad w=\left\{\begin{array}{l}
w_{1} \\
w_{2} \\
w_{3}
\end{array}\right\}
\end{aligned}
$$

\section{B. Equations of Motion}

The equations of motion of the linear elastic domain are given by the extended Hamilton's principle applied in a time interval $\left[t_{1}, t_{2}\right]$ and in the spatial domain $\Omega$ as

$$
\int_{t_{1}}^{t_{2}}\left\{\int_{\Omega}[\delta(\mathcal{K}-u)+\overline{\delta \mathcal{W}}] \mathrm{d} \mathbf{x}\right\} \mathrm{d} t=\overline{\delta \mathcal{A}}
$$

where $\overline{\delta \mathcal{W}}$ is the virtual work per unit volume of the external forces, $\overline{\delta \mathcal{A}}$ includes any virtual action on the boundaries of the domain $\Omega$ and at the ends of the time interval, and $\mathcal{K}$ and $u$ are the kinetic and strain energy densities, respectively. Overbars on virtual magnitudes indicate that they do not correspond to the variation of a function. Both energy densities are given by $\mathcal{K}=\frac{1}{2} \rho \dot{\mathbf{X}} \cdot \dot{\mathbf{X}}$ and $u=\frac{1}{2} \Gamma \cdot \mathbf{c} \cdot \Gamma$, respectively, where $\rho$ is the material density and $\mathbf{c}$ is the fourth-order tensor of elastic material constants (compliances). $\Gamma$ is the local Jaumann-Biot-Cauchy strain tensor given in a mixed-bases projection, as in Danielson and Hodges [25],

$$
\Gamma_{i j}=\frac{1}{2}\left(A_{i j}+A_{j i}\right)-\delta_{i j}, \quad \text { with } \quad A_{i j}=\mathbf{B}_{\mathbf{i}} \cdot \frac{\partial \mathbf{X}}{\partial \mathbf{x}} \cdot \mathbf{b}_{\mathbf{j}}
$$

Spatial differentiation of Eq. (4) gives the local strain tensor in the solid as

$$
\begin{aligned}
\Gamma & =\Gamma_{\gamma} \gamma(x, t)+\Gamma_{\kappa} \kappa(x, t)+\Gamma_{\mathbf{q}_{\mathbf{n}}} q_{n}(x, t)+\Gamma_{\mathbf{q}_{\mathbf{n}}^{\prime}} q_{n}^{\prime}(x, t) \\
& +\Gamma_{\mathbf{w}} w+\Gamma_{\mathbf{l}} w^{\prime}
\end{aligned}
$$

where $\Gamma_{(\bullet)}$ are a set of linear operators defined as in Cesnik and Hodges [4] , and $\gamma$ and $\kappa$ are the column matrix form of the force and moment generalized strains, respectively, defined as [20]

$$
\gamma=\left\{\begin{array}{c}
\gamma_{11} \\
2 \gamma_{12} \\
2 \gamma_{13}
\end{array}\right\}=C^{B a} R_{a}^{\prime}-C^{b a} r_{a}^{\prime}, \quad \kappa=\left\{\begin{array}{c}
\kappa_{1} \\
\kappa_{2} \\
\kappa_{3}
\end{array}\right\}=K_{B}-k_{b}
$$

Analogously, time differentiation of Eq. (4) defines the local velocities in the solid. The local inertial velocity vector $\dot{\mathbf{X}}$ is obtained as

$$
\dot{\mathbf{X}}=\mathbf{V}+\boldsymbol{\Omega} \times\left(x_{\alpha} \mathbf{B}_{\alpha}+\boldsymbol{\Psi}_{\mathbf{n}} q_{n}+\mathbf{w}\right)+\left(\boldsymbol{\Psi}_{\mathbf{n}} \dot{q}_{n}+\dot{\mathbf{w}}\right)
$$

where the components of the translational, $\mathbf{V}=V_{B, i} \mathbf{B}_{\mathbf{i}}$, and rotational, $\boldsymbol{\Omega}=\Omega_{B, i} \mathbf{B}_{\mathbf{i}}$, velocity vectors (defined with respect to an inertial reference frame) are given by the following kinematical relations: 


$$
V_{B}=C^{B a}\left(\dot{R}_{a}+\tilde{\omega}_{a} R_{a}+v_{a}\right), \quad \tilde{\Omega}_{B}=-\dot{C}^{B a} C^{a B}+C^{B a} \tilde{\omega}_{a} C^{a B}
$$

Subindexes in the vectors refer to the reference system in which their components are known. Substituting the strain and velocity fields of Eqs. (10) and (12), respectively, into the strain and kinetic energy densities, the variational problem of Eq. (8) defines without any approximation the dynamics of a slender solid in two sets of variables: 1) the averaged variables along the reference line $\left\{\mathbf{R}, \mathbf{C}^{\mathbf{B a}}, q_{n} ; \gamma, \mathbf{\kappa}, q_{n}^{\prime} ; \mathbf{V}, \boldsymbol{\Omega}, \dot{q}_{n}\right\}$, which are only spatial functions of the longitudinal coordinate, $x$, and are thus the long-scale variables; and 2) the residual local warping field and its derivatives $\left\{\mathbf{w}, \mathbf{w}^{\prime}, \dot{\mathbf{w}}\right\}$, which will be referred to as small-scale variables.

The condition of slenderness, $h / L \ll 1$, ensures that the energy contributions of these small-scale variables in the three-dimensional dynamic Eq. (8) will be small compared to those of the long-scale variables, and so it effectively defines a multiscale problem in the slender solid. As a result, one can find an asymptotic approximation to the variational three-dimensional problem by successively solving the problem at the different scales. This methodology is known as the variational-asymptotic method [3] , on which the book by Le [ㅁ] provides a good introduction.

\section{Cross-Sectional Analysis}

The solution to the cross-sectional (small-scale) problem in the present scope was presented in [15]. It is assumed that the contributions of $\dot{\mathbf{w}}$ and $\boldsymbol{\Omega} \times\left(\boldsymbol{\Psi}_{\mathrm{n}} q_{n}+\mathbf{w}\right)$ to the kinetic energy are negligible, which effectively reduces the small-scale problem to the minimization of the strain energy density at each cross section as function of the (small) local warping displacements. The small-scale problem becomes a constrained minimization problem, defined as

$$
\delta \int_{A(x)}\left[u\left(\hat{\gamma}, \hat{\kappa}, \hat{q}_{n}, \hat{q}_{n}^{\prime} ; w, w^{\prime}\right)+\lambda \bar{\Psi} \cdot w\right] \mathrm{d} A=0
$$

where the symbol $\hat{\bullet}$ was introduced to denote the prescribed variables in the cross-sectional problem and $\lambda$ are the Lagrange multipliers associated with the orthogonality constraints imposed by Eq. (5). A detailed analysis of the different contributions in Eq. (14) leads to the identification of different orders in the equation (i.e., $h / L,(h / L)^{2}$, etc.) and a general solution to this problem is obtained by a finiteelement discretization of the cross section. The first-order solution results in [15]

$$
\begin{aligned}
& w\left(x_{1}, x_{2}, x_{3}\right)=w_{\gamma}\left(x_{2}, x_{3}\right) \hat{\gamma}\left(x_{1}\right)+w_{\kappa}\left(x_{2}, x_{3}\right) \hat{\kappa}\left(x_{1}\right) \\
& \quad+w_{q_{n}}\left(x_{2}, x_{3}\right) \hat{q}_{n}\left(x_{1}\right)+w_{q_{n}^{\prime}}\left(x_{2}, x_{3}\right) \hat{q}_{n}^{\prime}\left(x_{1}\right)+\text { HOT }
\end{aligned}
$$

where HOT is higher-order terms.

Therefore, as a result of this linear optimal problem, one obtains a matrix of first-order warping influence coefficients, $\left[\begin{array}{llll}w_{\gamma} & w_{\kappa} & w_{q_{n}} & w_{q_{n}^{\prime}}\end{array}\right]$. The strain energy density can be explicitly integrated at each cross section to define the strain energy per unit length of the beam as

$$
\mathcal{U}=\int_{A(x)} u \mathrm{~d} A=\frac{1}{2}\left\{\begin{array}{llll}
\gamma^{T} & \kappa^{T} & q^{T} & q^{\prime T}
\end{array}\right\}[S]\left\{\begin{array}{c}
\gamma \\
\kappa \\
q \\
q^{\prime}
\end{array}\right\}+\text { HOT }
$$

where the constant matrix $[S]$ is the first-order asymptotic approximation to the stiffness matrix. Higher-order approximations were presented in [15]. To complete the solution of the crosssectional problem, the kinetic energy density needs to be evaluated using the decomposition of the velocity field given by Eq. (12). With the assumption of negligible contributions of $\dot{\mathbf{w}}$ and $\boldsymbol{\Omega} \times\left(\boldsymbol{\Psi}_{\mathbf{n}} q_{n}+\mathbf{w}\right)$, this task is straightforward, and defines the kinetic energy per unit length as

$$
\mathcal{K}=\int_{A} \mathcal{K} \mathrm{d} A=\frac{1}{2}\left\{\begin{array}{lll}
V_{B}^{T} & \Omega_{B}^{T} & \dot{q}_{n}^{T}
\end{array}\right\}[M]\left\{\begin{array}{c}
V_{B} \\
\Omega_{B} \\
\dot{q}_{n}
\end{array}\right\}
$$

where the constant matrix $[M]$ is the inertia matrix for the cross section.

\section{One-Dimensional Analysis at the Reference Line}

The small-scale cross-sectional problem has defined the homogenization constants for the cross section, and the problem has been effectively reduced to the analysis of the evolution of averaged variables along the reference line. This one-dimensional long-scale problem is now set up in a continuous segment of arc length $l$ of the reference line, which will be referred to as a structural member. The solution procedure follows the one developed by Hodges [20]. From Eqs. (16) and (17), the left-hand side terms in Eq. () can be integrated at each cross section as

$$
\overline{\delta \pi} \equiv \int_{0}^{l}[\delta(\mathcal{K}-\mathcal{U})+\overline{\delta \mathcal{W}}] \mathrm{d} x
$$

where $\overline{\delta \pi}$ is the virtual total potential and $\overline{\delta \mathcal{W}}$ is the virtual work per unit length of the applied loads, which is decomposed in the virtual work per unit length of the structural damping forces and of the applied external forces, $\overline{\delta \mathcal{W}_{d}}$ and $\overline{\delta \mathcal{W}_{e}}$, respectively. To evaluate the different terms in Eq. (18), one needs to select first an irreducible (as defined in [26]) set of independent variables to obtain variations of the functionals. Six independent variables (three displacements, $u$, and three rotations, $\varphi$, of the reference frame $a$ ) determine the rigidbody motion of the member. Additional independent variables account for the deformations of the flexible structure: the set of displacements and rotations along the reference line, as well as the amplitudes of the finite-section modes.

\section{Strain Energy per Unit Length}

In Eq. (16) the strain energy per unit length was approximated as a quadratic functional of the form $\mathcal{U}=\mathcal{U}\left[\gamma(x), \kappa(x), q(x), q^{\prime}(x)\right]$. The partial derivatives of the strain energy are identified as section stress resultants

$$
\begin{aligned}
F_{B} & =\left(\frac{\partial \mathcal{U}}{\partial \gamma}\right)^{T}, & M_{B} & =\left(\frac{\partial \mathcal{U}}{\partial \kappa}\right)^{T} \\
Q_{s_{0}} & =\left(\frac{\partial \mathcal{U}}{\partial q}\right)^{T}, & Q_{s_{1}} & =\left(\frac{\partial \mathcal{U}}{\partial q^{\prime}}\right)^{T}
\end{aligned}
$$

where $F_{B}$ and $M_{B}$ can be identified as the internal force and moment column vectors, whereas the $Q_{s}$ terms are the conjugate forces corresponding to the deformation of the structure in the finite-section modes. The variation of the internal energy density is given by

$$
\delta \mathcal{U}=\delta \gamma^{T} F_{B}+\delta \kappa^{T} M_{B}+\delta q^{T} Q_{s_{0}}+\delta q^{\prime T} Q_{s_{1}}
$$

The column vector of virtual rotations from frame $a$ to $B$, expressed in frame $a$, is defined as $\tilde{\delta \Phi}_{a}=\delta C^{a B} C^{B a}$. The virtual straindisplacement relations are obtained from Eq. (11) as

$$
\begin{gathered}
\delta \gamma=C^{B a}\left(\frac{\mathrm{d}}{\mathrm{d} x} \delta R_{a}+\tilde{R}_{a}^{\prime} \overline{\delta \Phi}_{a}\right)=C^{B a} \frac{\mathrm{d}}{\mathrm{d} x} \delta R_{a}+\left(\tilde{\gamma}+\tilde{e}_{1}\right) C^{B a} \overline{\delta \Phi}_{a} \\
\delta \kappa=C^{B a} \frac{\mathrm{d}}{\mathrm{d} x} \overline{\delta \Phi}_{a}, \quad \delta q^{\prime}=\frac{\mathrm{d}}{\mathrm{d} x} \delta q
\end{gathered}
$$

\section{Kinetic Energy per Unit Length}

Homogenization of the cross-sectional inertia properties in Eq. (17) approximates the kinetic energy per unit length as a bilinear functional of the form $\mathcal{K}=\mathcal{K}\left[V_{B}(x), \Omega_{B}(x), \dot{q}(x)\right]$. The partial derivatives of the kinetic energy define the section momentum resultants

$$
P_{B}=\left(\frac{\partial \mathcal{K}}{\partial V_{B}}\right)^{T}, \quad H_{B}=\left(\frac{\partial \mathcal{K}}{\partial \Omega_{B}}\right)^{T}, \quad Q_{t}=\left(\frac{\partial \mathcal{K}}{\partial \dot{q}}\right)^{T}
$$


momentum column vectors, whereas $Q_{t}$ are the generalized inertia momenta associated with the finite-section modes. From the previous definitions, the variation of the kinetic energy density is

$$
\delta \mathcal{K}=\delta V_{B}^{T} P_{B}+\delta \Omega_{B}^{T} H_{B}+\delta \dot{q}^{T} Q_{t}
$$

The virtual quantities in $(\underline{23})$ are now expressed as a function of the independent variables as

$$
\begin{aligned}
\delta V_{B} & =C^{B a} \delta v_{a}-C^{B a} \tilde{R}_{a} \delta \omega_{a}+C^{B a}\left(\frac{\mathrm{d}}{\mathrm{d} t} \delta R_{a}+\tilde{\omega}_{a} \delta R_{a}\right) \\
& +\tilde{V}_{B} C^{B a} \overline{\delta \Phi}_{a} \\
\delta \Omega_{B} & =C^{B a} \delta \omega_{a}+C^{B a}\left(\frac{\mathrm{d}}{\mathrm{d} t} \overline{\delta \Phi}_{a}+\tilde{\omega}_{a} \overline{\delta \Phi}_{a}\right), \quad \delta \dot{q}=\frac{\mathrm{d}}{\mathrm{d} t} \delta q
\end{aligned}
$$

where the first two equations were obtained by taking variations in Eq. (13). The variations of the rigid-body virtual velocities and displacements in the member frame can be expressed as

$$
\delta v_{a}=\delta \dot{u}_{a}+\tilde{\omega}_{a} \delta u_{a}, \quad \delta \omega_{a}=\overline{\delta \dot{\varphi}}_{a}+\tilde{\omega}_{a} \overline{\delta \varphi}_{a}
$$

\section{Virtual Work per Unit Length of the External Forces}

Consider the vector $\mu$ of distributed forces per unit volume applied on the three-dimensional solid. The virtual work per unit length of the reference line produced by this force is given by multiplying it by the virtual displacement with respect to an inertial frame (the global frame $o$ ), as

$$
\overline{\delta \mathcal{W}_{e}}=\int_{A(x)} \boldsymbol{\mu} \cdot(\boldsymbol{\delta} \mathbf{X}+\delta \mathbf{u}) \mathrm{d} A
$$

The position vector $\mathbf{X}$ is given in Eq. (4) as a function of the deformation of the reference line and the cross-sectional warping. The latter can be approximated by the warping influence coefficients obtained in Eq. (15) to give

$$
X_{o}=C^{o a} R_{a}+C^{o B}\left(\xi+\Psi_{q}+w_{\chi} \chi\right)
$$

where $\xi^{T}=\left\{\begin{array}{lll}0 & x_{2} & x_{3}\end{array}\right\}$ contains the cross-sectional coordinates,

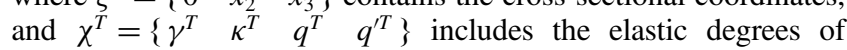
freedom. The corresponding virtual displacements are

$$
\begin{aligned}
& \delta X_{o}=-C^{o a} \tilde{X}_{a} \overline{\delta \varphi}_{a}+C^{o a} \delta R_{a}-C^{o B}\left(\tilde{\xi}+\tilde{\Psi}_{q}+\tilde{w}_{, \chi} \chi\right) C^{B a} \overline{\delta \Phi}_{a} \\
& \quad+C^{o B}\left(\Psi \delta q+w_{\chi} \delta \chi\right)
\end{aligned}
$$

Substituting this expression in Eq. (26), one obtains the virtual work per unit length of the applied forces as

$$
\begin{aligned}
& {\overline{\delta \mathcal{W}_{e}}}_{e}=\delta u_{a}^{T} C^{a B} f_{0}+\overline{\delta \varphi}_{a}^{T} C^{a B} m_{0}^{*}+\delta R_{a}^{T} C^{a B} f_{0}+\delta R_{a}^{\prime T} C^{a B} f_{1} \\
& +\overline{\delta \Phi}_{a}^{T} C^{a B} m_{0}+\overline{\delta \Phi^{\prime}}{ }_{a}^{T} C^{a B} m_{1}+\delta q^{T} f_{s_{0}}+\delta q^{\prime T} f_{s_{0}}
\end{aligned}
$$

The following applied forces per unit length have been used in this expression:

$$
\begin{gathered}
f_{0}=\int_{A(x)} \mu_{B} \mathrm{~d} A, \quad f_{s_{0}}=\int_{A(x)}\left(\Psi^{T}+w_{q}^{T}\right) \mu_{B} \mathrm{~d} A \\
m_{0}^{*}=\int_{A(x)} C^{B a} \tilde{X}_{a} C^{a B} \mu_{B} \mathrm{~d} A, \quad m_{0}=m_{0}^{*}-C^{B a} \tilde{R}_{a} C^{a B} f_{0} \\
f_{1}=\int_{A(x)} w_{\gamma}^{T} \mu_{B} \mathrm{~d} A, \quad f_{s_{1}}=\int_{A(x)} w_{q^{\prime}}^{T} \mu_{B} \mathrm{~d} A \\
m_{1}=\int_{A(x)} w_{\kappa}^{T} \mu_{B} \mathrm{~d} A
\end{gathered}
$$

where $\mu_{B}$ is the projection of the distributed force $\mu$ onto the deformed reference, $B$. From Eqs. (27) and (30), the virtual work of the external forces depends on the actual deformation through the cross-sectional warping field. The applied work also includes the contribution from the resultant applied forces per unit length conjugated of the finite-section modes $f_{s_{0}}$ and its longitudinal derivatives, $f_{s_{1}}$.

If cross sections are rigid and the motion of the member reference frame ( $a$ ) is prescribed, that is, $\delta u_{a}=\overline{\delta \varphi_{a}}=0$, then the only nonzero terms in Eq. (30) are the classical beam forces and moments:

$$
f_{0, \mathrm{cl}}=f_{0}=\int \mu_{B} \mathrm{~d} A, \quad m_{0, \mathrm{cl}}=\int \tilde{\xi} \mu_{B} \mathrm{~d} A
$$

\section{Virtual Work per Unit Length of the Structural Damping Forces}

Structural damping is modeled here by forces/moments per unit length proportional to the rate of change of the generalized internal forces (expressed in their components in the deformed frame) at each cross section, that is,

$$
\left.\left\{\begin{array}{c}
f_{d 0 B} \\
m_{d 0 B} \\
f_{s d_{0}} \\
f_{s d_{1}}
\end{array}\right\}=-g \frac{\mathrm{d}}{\mathrm{d} t}\left\{\begin{array}{c}
F_{B} \\
M_{B} \\
Q_{s_{0}} \\
Q_{s_{1}}
\end{array}\right\}=-\left[\begin{array}{cccc}
g_{F} & 0 & 0 & 0 \\
0 & g_{M} & 0 & 0 \\
0 & 0 & g_{s_{0}} & 0 \\
0 & 0 & 0 & g_{s_{1}}
\end{array}\right] \mathrm{d} \frac{\mathrm{d} t}{M_{B}} \begin{array}{c}
F_{B} \\
Q_{s_{0}} \\
Q_{s_{1}}
\end{array}\right\}
$$

where $g$ is a diagonal matrix of nonnegative structural damping coefficients. The virtual work of these forces is given by

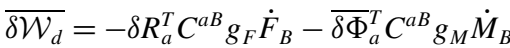

$$
\begin{aligned}
& -\delta q^{T} g_{s_{0}} \dot{Q}_{s_{0}}-\delta q^{T} g_{s_{1}} \dot{Q}_{s_{1}}
\end{aligned}
$$

\section{Intrinsic Equations in the Member Frame}

The strain energy (20), kinetic energy (23), and virtual work (29) and (33) are substituted into the expression of the total potential per unit length (18). Through integration by parts in both time and space, one obtains the geometrically nonlinear intrinsic equilibrium equations at the reference line. In strong form, they are written as

$$
\begin{gathered}
\left(\frac{\mathrm{d}}{\mathrm{d} t}+\tilde{\Omega}_{B}\right) P_{B}=\left(\frac{\mathrm{d}}{\mathrm{d} x}+\tilde{K}_{B}\right)\left(F_{B}-f_{1}\right)-g_{F} \frac{\mathrm{d}}{\mathrm{d} t} F_{B}+f_{0} \\
\left(\frac{\mathrm{d}}{\mathrm{d} t}+\tilde{\Omega}_{B}\right) H_{B}+\tilde{V}_{B} P_{B}=\left(\frac{\mathrm{d}}{\mathrm{d} x}+\tilde{K}_{B}\right)\left(M_{B}-m_{1}\right) \\
+\left(\tilde{e}_{1}+\tilde{\gamma}\right) F_{B}-g_{M} \frac{\mathrm{d}}{\mathrm{d} t} M_{B}+m_{0} \\
\frac{\mathrm{d}}{\mathrm{d} t} Q_{t}=\frac{\mathrm{d}}{\mathrm{d} x}\left(Q_{s_{1}}+g_{s_{1}} \frac{\mathrm{d}}{\mathrm{d} t} Q_{s_{1}}-f_{s_{1}}\right)-\left(Q_{s_{0}}+g_{s_{0}} \frac{\mathrm{d}}{\mathrm{d} t} Q_{s_{0}}-f_{s_{0}}\right)
\end{gathered}
$$

If the rigid-body motion of the member is unconstrained, the system is completed by the free-body equations given in the member frame

$$
\left(\frac{\mathrm{d}}{\mathrm{d} t}+\tilde{\omega}_{a}\right) p_{a}=\int_{0}^{l} C^{a B} f_{0} \mathrm{~d} x, \quad\left(\frac{\mathrm{d}}{\mathrm{d} t}+\tilde{\omega}_{a}\right) h_{a}=\int_{0}^{l} C^{a B} m_{0}^{*} \mathrm{~d} x
$$

where two new aggregate vector magnitudes were defined to describe the free-body motion of the member: $\mathbf{p}$, the member translational momentum, and $\mathbf{h}$, the member angular momentum about the origin of the member reference frame, $a$. Their components in the $a$ frame are given by

$$
p_{a}=\int_{0}^{l} C^{a B} P_{B} \mathrm{~d} x, \quad h_{a}=\int_{0}^{l}\left(C^{a B} H_{B}+\tilde{R}_{a} C^{a B} P_{B}\right) \mathrm{d} x
$$

Equations (35) are the expression for an elastic system of the Euler-Lagrange rigid-body equations. Note that both Eqs. (34) and (35) are given in their intrinsic form, that is, the local elastic equilibrium Eqs. (34) are given in the local deformed frame, $B$, and 
the global dynamic equilibrium Eqs. (35) are given in the member frame, $a$ (a body-fixed representation, if frame $a$ is forced to move with the member). Three different extensions can be identified in this formulation when compared to the original intrinsic theory of moving beams of Hodges [20]. First, the motion of the reference frame is brought into the system by including Eqs. (35). Second, the addition of the finite-section deformation modes into the onedimensional representation of the structure brings an additional differential equation for each mode into the final system, Eqs. (34). And third, a more general definition of the forcing terms in the onedimensional equations is introduced in Eqs. (30) by using the actual structural deformation (defined by a set of warping influence coefficients in the cross-sectional analysis) in the computation of the virtual work of the external forces.

\section{Mixed-Form Finite-Element Solution}

A numerical solution is proposed based on a mixed (or hybrid) form of the equations on the reference line. The procedure is very similar to the one introduced by Hodges et al. [21] and it will not be fully developed here. Basically, the weak form of the equilibrium Eqs. (34) and (35) is augmented imposing the kinematical relations (11) and (13) through Lagrange multipliers, which defines a generalized virtual potential $\delta \pi^{*}$. Rodrigues parameters [24], $\theta_{a}^{B}$, are used to parameterize the finite rotations between the member reference frame $(a)$ and the deformed frame $(B)$. Note that this parameterization already includes any initial twist and curvature of the reference line. The mixed form of Eqs. (34) and (35) defines an appropriate starting point for a (spatial) finite-element discretization of the one-dimensional dynamic equations. The discretization is defined in each member; next, members are assembled using the boundary and/or joint conditions at their ends. If free-body motions are unconstrained, the problem is completed by adding the rigidbody equations from Eqs. (35). The dependency with time is left explicitly and the original system of partial-differential equations is converted into a set of ordinary-differential/algebraic equations in time domain. The generalized virtual potential is discretized in $N$ elements at a given time as

$$
\overline{\delta \pi^{*}}=\sum_{e=1}^{N} \overline{\delta \pi_{e}^{*}}
$$

The length of the $e$ th element is $\Delta l_{e}$, and is bounded by nodes $e$ and $e+1$. One advantage of the mixed formulation is that one can select very simple shape functions in the element. Here, constantvalued functions are selected within the elements as

$$
\begin{gathered}
\left\{\begin{array}{c}
R_{a}(x) \\
\theta_{a}^{B}(x) \\
q(x)
\end{array}\right\}=\left\{\begin{array}{c}
R_{e} \\
\theta_{e} \\
q_{e}
\end{array}\right\} \\
\left\{\begin{array}{c}
F_{B}(x) \\
M_{B}(x) \\
Q_{s_{1}}(x)
\end{array}\right\}=\left\{\begin{array}{c}
F_{e} \\
M_{e} \\
Q_{s e}
\end{array}\right\}, \text { and }\left\{\begin{array}{c}
P_{B}(x) \\
H_{B}(x) \\
Q_{t}(x)
\end{array}\right\}=\left\{\begin{array}{c}
P_{e} \\
H_{e} \\
Q_{t e}
\end{array}\right\}
\end{gathered}
$$

with $x \in\left[x_{e}, x_{e+1}\right]$. This defines a $3 \times\left(6+N_{q}\right)$ element state vector, where $N_{q}$ is the number of finite-section modes, as

$$
\mathrm{X}_{e}^{T}(t)=\left[\begin{array}{lllllllll}
R_{e}^{T} & \theta_{e}^{T} & q_{e}^{T} & F_{e}^{T} & M_{e}^{T} & Q_{s e}^{T} & P_{e}^{T} & H_{e}^{T} & Q_{t e}^{T}
\end{array}\right]
$$

and a member state vector as

$$
\mathrm{X}^{T}(t)=\left[\begin{array}{lll}
\mathrm{X}_{1}^{T} & \cdots & \mathrm{X}_{N}^{T}
\end{array}\right]
$$

The boundary values of displacements and forces at $x=0$ and $x=l$ defined additional unknowns in the problem. Their components in the member reference frame $(a)$ define the member boundary state vector as

$$
\hat{\mathrm{X}}_{x=x_{0}}^{T}(t)=\left[\begin{array}{llllll}
\hat{R}_{x=x_{0}}^{T} & \hat{\theta}_{x=x_{0}}^{T} & \hat{q}_{x=x_{0}}^{T} & \hat{F}_{x=x_{0}}^{T} & \hat{M}_{x=x_{0}}^{T} & \hat{Q}_{s_{1}, x=x_{0}}^{T}
\end{array}\right]
$$

The total number of unknowns in the discretization of the structural member is $(3 \times N+4) \times\left(6+N_{q}\right)$. In general, all of them are functions of time. The former discretization allows the explicit integration in $x$ of Eq. (37), which can be written as a set of differential-algebraic equations in time domain for each member:

$$
A(\mathrm{X}) \cdot \dot{\mathrm{X}}=\mathrm{L}\left(\mathrm{X}, \hat{\mathrm{X}}_{x=0}, \hat{\mathrm{X}}_{x=l}\right)-\mathrm{S}\left(\mathrm{X}, \hat{\mathrm{X}}_{x=0}, \hat{\mathrm{X}}_{x=l}\right)
$$

where $A$ is the (singular) inertia matrix operator, $S$ is the structural column matrix operator, and $L$ is the load column matrix operator. These equations are complemented by $6+N_{q}$ additional equations on each member end, which can be defined either from external boundary conditions (such as clamped or free end) or from joint conditions between two or more members. For a cantilever beam, the boundary conditions would be

$$
K_{R} \hat{X}_{x=0}=0 \quad \text { and } \quad K_{F} \hat{X}_{x=l}=b
$$

with $K_{R}=\left[\begin{array}{ll}I & 0\end{array}\right], K_{F}=\left[\begin{array}{ll}0 & I\end{array}\right]$ being 0 and $I$ the zero and unit matrices of dimension $\left(6+N_{q}\right)$, respectively, and with $b$ being the vector of applied tip loads. Three different solution schemes were set up for Eq. (42): a time-domain solution, a steady-state solution, and a linearized vibration analysis. The time-domain solution uses an implicit three-point backwards Euler integration scheme with a variable time step, whereas the steady-state solution is obtained by setting $\dot{\mathrm{X}}=0$. In both cases, a bandwidth-optimized system of algebraic equations is obtained, which is solved for each time and load step, respectively, using a Newton-Raphson method with analytical Jacobians. For the linearized vibration solution, the dynamic Eqs. (34) are linearized around a nonlinear steady state and the complex-domain eigenvalues and eigenvectors from the resulting first-order system of equations is obtained using ARPACK [27].

\section{Numerical Examples}

Three numerical examples are proposed to illustrate the ability of the present theory to capture higher-order deformations with a beamlike solution framework. First, the effect of cross-sectional deformations is studied in the natural vibration modes of a simple isotropic thin strip. The second example is the computation of the local deformations in a cantilever box beam with embedded actuation. Distributed actuation generates local wall deformations, which are captured by the proposed theory through finite-section modes. Finally, the effect of cross-sectional deformations is studied on the linear vibration characteristics of the same box-beam configuration. Results compare a classical one-dimensional Timoshenko bending, extension, shear, twist (BEST) description with 6 degrees of freedom, the expanded one-dimensional model with finite-section modes, and plate finite-element solutions obtained using MSC.Nastran. The goal in this section is to show potential applications of the finite-section mode concept, which defines intrinsically a linearized problem at the small-scale (cross-sectional) level. To focus the reader on this aspect of the theory, numerical examples in this paper have been restricted to small displacements of the reference line, even though the proposed analysis is a geometrically nonlinear formulation.

\section{A. Isotropic Thin Strip}

Consider an isotropic thin strip $(E=1.0 \mathrm{GPa}, \quad v=0.3$, $\left.\rho=1000 \mathrm{~kg} / \mathrm{m}^{3}\right)$ of length-to-width ratio $L / h=4$ and crosssectional width-to-thickness ratio $h / t=50$. Thickness was chosen to be $t=2.79 \mathrm{~mm}$. For this configuration, linear vibration results are obtained by a finite-element one-dimensional model based on the present approach and compared to a shell model. In both cases, the discretization was refined until a desired convergence rate was achieved. In the one-dimensional model, the effect of camber 
Table 1 First natural frequencies (in $\mathrm{Hz}$ ) of the thin strip

\begin{tabular}{ccccccccc}
\hline \hline & \multicolumn{2}{c}{ Shell (two-dimensional) } & \multicolumn{2}{c}{ Timoshenko (one-dimensional) } & \multicolumn{2}{c}{ One-dimensional with camber bending } \\
\hline Type & No. & Freq. & No. & Freq. & MAC & No. & Freq. & MAC \\
\hline VB1 & 1 & 1.469 & 1 & 1.457 & 1.000 & 1 & 1.470 & 1.000 \\
VB2 & 2 & 9.191 & 2 & 9.133 & 1.000 & 2 & 9.209 & 1.000 \\
T1 & 3 & 11.80 & 3 & 11.05 & 0.998 & 3 & 11.05 & 0.998 \\
VB3 & 4 & 25.80 & 4 & 25.58 & 1.000 & 4 & 25.85 & 1.000 \\
T2 & 5 & 36.43 & 5 & 33.14 & 0.989 & 5 & 33.14 & 0.989 \\
VB4 & 6 & 50.73 & 6 & 50.17 & 0.999 & 6 & 50.82 & 1.000 \\
T3 & 7 & 63.97 & 7 & 55.24 & 0.971 & 7 & 55.25 & 0.971 \\
LB1 & 8 & 69.28 & 8 & 71.62 & 1.000 & 8 & 71.62 & 1.000 \\
VB5 & 9 & 84.08 & 10 & 83.00 & 0.996 & 10 & 84.24 & 1.000 \\
T4 & 10 & 96.02 & 9 & 77.37 & 0.944 & 9 & 77.37 & 0.944 \\
\hline \hline
\end{tabular}

bending is included by a finite-section mode, defined as

$$
\Psi\left(x_{2}, x_{3}\right)=\left\{\begin{array}{lll}
0 & 0 & \left(\frac{2 x_{2}}{h}\right)^{2}-\frac{1}{3}
\end{array}\right\}^{T}
$$

where the reference for the cross-sectional coordinates was selected at the area centroid of the cross section. This definition satisfies the orthogonality conditions of Eq. (6). The converged natural frequencies (in $\mathrm{Hz}$ ) of the first 10 vibration modes of this configuration are included in Table 1 . They are identified as vertical bending (VB) or lateral bending (LB) modes or twist modes (T). The eigenvectors obtained by both approximations are compared using the modal assurance criteria (MAC) number (i.e., the normalized scalar product of both mode shapes).

From these results, it should be noted first that vertical bending modes compare very well. In particular, the mode shapes obtained by the one-dimensional formulations capture the local warping effects, as seen in the fifth bending mode in Fig. 2. It should be noted that the beam model using the 6-degrees-of-freedom (DOF) Timoshenko description does capture the cross-sectional deformation through the warping influence coefficients corresponding to the bending curvature [i.e., second column of matrix $w_{\kappa}$, defined in Eq. (15)], whereas the model with the camber-bending finite-section mode captures the warping through the additional degree of freedom. There is, however, a small difference between these two results that can be observed at both root and tip of the strip; the bending curvature is $\kappa_{2} \neq 0$ at the root and $\kappa_{2}=0$ at the tip, and so it is the corresponding warping at those cross sections in the model with the Timoshenko DOF. When the camber bending is explicitly included in the model, cantilever conditions yield $q=0$ at the root and $q^{\prime}=0$ at the tip, which is closer to the behavior of the shell model.

In Table 1, the comparison between both one-dimensional models and the shell model for the natural frequencies of the lateral bending and twist modes shows a larger error, which increases with frequency. This is particularly critical for the torsional modes, for which the camber-bending deformation does not improve the model accuracy. The situation is analogous to the aforementioned one for the root warping with the Timoshenko DOF but for the torsional curvature, $\kappa_{1}$. The beam models in this work do not enforce $\kappa_{1}=0$ at the root, and so it does not constrain the warping at the root. This situation would be solved by a generalized Vlasov model (see, for instance, [24]), which has not been included in the present analysis. At higher frequencies, some camber-bending-dominated modes start
Table 2 Thin strip: natural frequencies (in $\mathrm{Hz}$ ) of the first camber-bending-dominated vibration modes

\begin{tabular}{ccccc}
\hline \hline \multicolumn{2}{c}{ Shell (2-D) } & \multicolumn{3}{c}{ 1-D with camber } \\
\hline No. & Freq. & No. & Freq. & MAC \\
\hline 17 & 196.1 & 20 & 199.6 & 0.991 \\
19 & 230.9 & 22 & 227.5 & 0.962 \\
21 & 276.2 & 26 & 261.6 & 0.970 \\
\hline \hline
\end{tabular}

to appear. They would not be captured by a classical (EulerBernoulli or Timoshenko) beam model, and a finite-section mode for the camber-bending deformation is then required. The frequencies of the first natural vibration modes that can be identified as camberbending dominated are included in Table 2 . The eigenvectors for the first and third camber-bending-dominated mode are shown in Figs. 3 and 4 , respectively. They were computed from models with $4 \overline{0}$ elements in the longitudinal direction.

As it can be observed, the additional degree of freedom in the onedimensional model has captured quite accurately the lowest camber vibration modes of the thin strip. For shorter plates, higher-order camber deformations could be included by defining additional finitesection modes.

\section{B. Static Response of Composite Box Beam with Embedded Actuation}

This second numerical example corresponds to a cantilever composite box beam with midwall dimensions $20 a$ (length) $\times$ $2 a$ (width) $\times a$ (height) and with embedded piezoelectric actuators. Each wall is made of four orthotropic plies of thickness $a / 100$. This configuration was first introduced in [15]. A dominant dimension can be identified in the structure and $\mathrm{a}$ one-dimensional model is

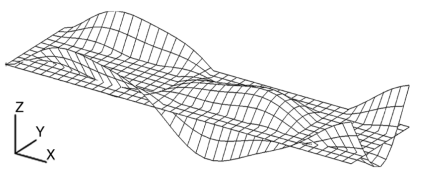

Shell Model

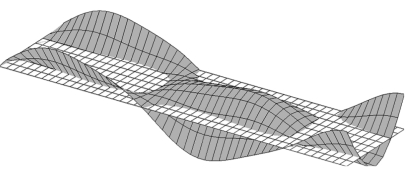

1-D with camber bending
Fig. 3 First camber-bending-dominated mode shape of the thin strip.

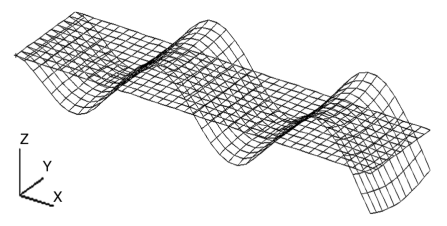

Shell Model

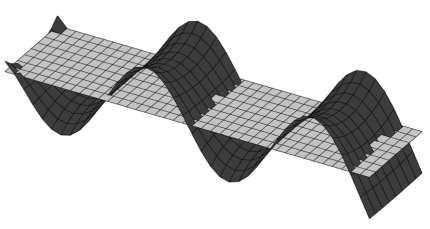

Timoshenko 1-D

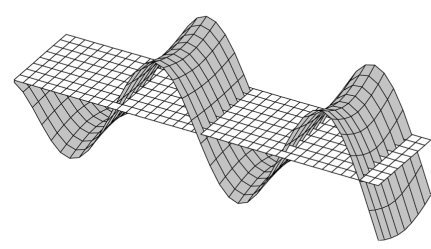

1-D with camber bending

Fig. 2 Comparison of fifth bending mode obtained with shell and one-dimensional models. 


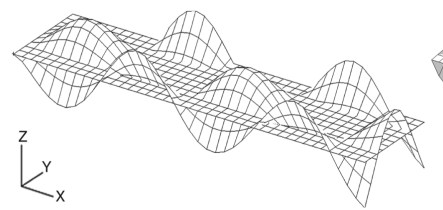

Shell Model

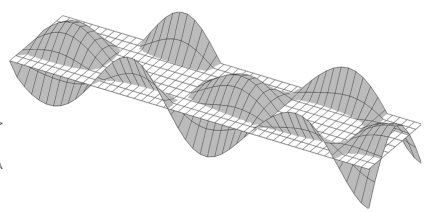

1-D with camber bending
Fig. 4 Third camber-bending-dominated mode shape of the thin strip.

proposed for its analysis, with the reference line passing through the area centroid of the cross sections. The thin-wall construction, however, implies the likely appearance of platelike components in the deformation field, which were found to be particularly important in two situations: 1) when the excitation comes from a distributed loading, such as the one generated by embedded piezoelectric actuators; and 2) with dynamic excitation at low-to-moderate frequencies.

Material elastic constants are $\mathrm{E}_{33}=\mathrm{E}_{22}=\mathrm{E}_{11} / 20, \mathrm{G}_{12}=\mathrm{G}_{13}=$ $\mathrm{E}_{11} / 10, \mathrm{G}_{23}=\mathrm{G}_{12} / 2, v_{12}=v_{13}=0.35$, and $v_{23}=0.4$ (with 1 in the direction of the fiber, 2 in the plane of the plies, and 3 through the thickness). All elastic plies are assumed to have piezoelectric properties. For the piezoelectric actuation response, a prescribed through-the-thickness constant electric field $E_{3}$ is assumed on the actuated plies, such that the free-strain deformation of a single ply is given by $d_{3,11} E_{3}=\Gamma_{11}^{o}$ in the direction of the fiber and $d_{3,22}=$ $-d_{3,11} / 3$ in the transverse direction. This characterization of the electric field corresponds to the usual assumption for the analysis of piezoelectric actuators in structural applications [28]. Two lay ups with several actuation architectures are studied (see Table 3), in which plies are numbered inside out and positive material orientation angle is defined in the clockwise direction.

For the numerical analyses in this section, consider $a=0.5 \mathrm{~m}$, $E_{11}=10^{7} \mathrm{~Pa}, \Gamma_{11}^{o}=300 \mu \varepsilon$. The present one-dimensional model is compared to a (two-dimensional) composite shell finite-element model with a similar discretization. Consider first results of a onedimensional model using the Timoshenko BEST description. Note that, although in thin-wall beams shear relief effects are small, transverse shear effects are still necessary to account for possible net shear actuation forces. The resulting static displacements $\left(u_{i}\right)$ and rotations $\left(\theta_{i}\right)$ at the beam midpoint $\left(x_{1}=10 a\right)$ for the different actuator configurations defined in Table $\underline{3}$ are shown in Table 4 . Displacements obtained by the one-dimensional beam model are compared to the kinematical interpolation of the shell displacement

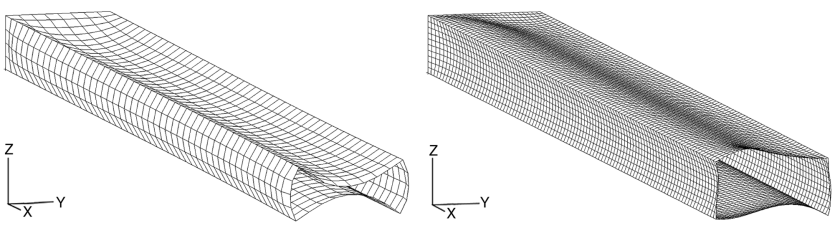

Case A3

Case A4
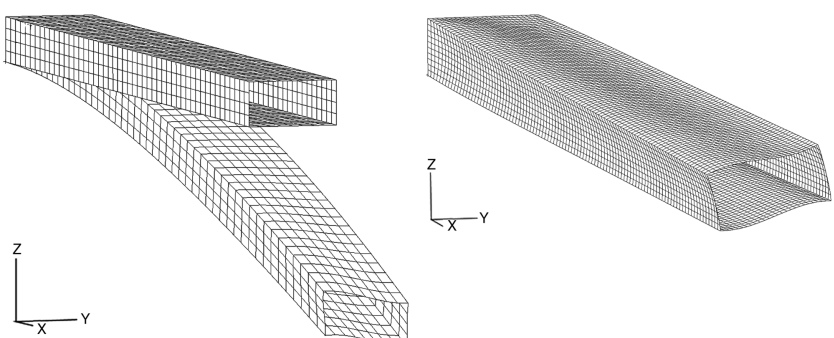

-

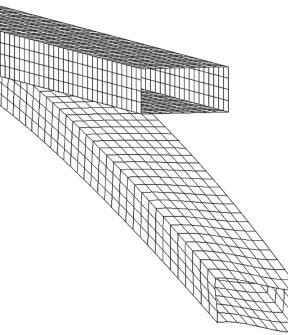

Case S3

Case S4

Fig. 5 Displacement field of shell model under active static loading (displacements magnified by 100).

field at the reference line. Figure 5 includes the actual displacements on the shell model for those cases in which local effects become more important.

In most situations the three-dimensional displacement field induced by the embedded actuators can be easily identified as beam deformations; that is the case for configurations $\mathrm{S} 1$ (transverse shear), S2 (extension), S3 (twist/bending), A1 (bending), and A2 (twist). However, in some cases local wall deformations are important and the Timoshenko DOF do not capture even the average motion of the structure. As proposed here, the one-dimensional model is expanded with a set of finite-section modes, which accounts for the local deformations of the beam walls. For the box beam under consideration, they are defined by harmonic functions at the walls, $\Psi_{n}=\cos \left(k \pi x_{\alpha} / L_{\alpha}\right)\left(L_{\alpha}\right.$ is the wall length and $k=1,2$. The actual amplitude of $\psi_{q}$ does not affect the results). They define a unique expansion of the cross-sectional warping field. The six modes in Fig. 6 are included in the proposed one-dimensional model. It should be remarked that the actual warping field associated with each finitesection mode is obtained through the minimization of the crosssectional strain energy. The resulting displacement field associated with each finite-section mode is obtained by adding the assumed

Table 3 Lay up and electric actuation for box beam-

\begin{tabular}{ccc}
\hline \hline Case & Elastic ply up & Electric actuation \\
\hline S1 & & $\left(0_{4}\right) \Uparrow \Downarrow ;(0 /+/ 0 /-) \Rightarrow \Leftarrow$ \\
S2 & $\left(45_{2} /-45_{2}\right) \Uparrow \Rightarrow ;\left(-45_{2} / 45_{2}\right) \Downarrow \Leftarrow$ & $(0 /+/+/ 0) \Uparrow \Downarrow \Rightarrow \Leftarrow$ \\
S3 & & $(0 /+/ 0) \Uparrow \Leftarrow ;(0 /-/-/ 0) \Downarrow \Rightarrow$ \\
S4 & & $(+/+)(-/ 0 / 0 /+) \Rightarrow ;(+/ 0 / 0 /-) \Leftarrow$ \\
A1 & & $(+/+) \Rightarrow ;(-/-/-/-) \Leftarrow$ \\
A2 & $\left(45 /-45_{2} / 45\right) \Uparrow \Downarrow \Rightarrow \Leftarrow$ & $(-/ 0 / 0 /+) \Rightarrow \Leftarrow$ \\
A3 & & $(-/ 0 / 0 /+) \Rightarrow ;(+/ 0 / 0 /-) \Leftarrow$ \\
A4 & & \\
\hline \hline
\end{tabular}

a $\Uparrow$ upper, $\Downarrow$ bottom, $\Rightarrow$ right, $\Leftarrow$ left wall; plies numbered inside out

Table 4 Displacements and rotations at center of the reference line $(x=10 a)$ under static actuation

\begin{tabular}{cccccccc}
\hline \hline Case & Model & $u_{1}, \mathrm{~mm}$ & $u_{2}, \mathrm{~mm}$ & $u_{3}, \mathrm{~mm}$ & $\theta_{1}, \mathrm{deg}$ & $\theta_{2}, \operatorname{deg}$ & $\theta_{3}, \operatorname{deg}$ \\
\hline S1 & 2-D/1-D & - & $0.02 / 0.03$ & $-1.42 /-1.39$ & - & - & - \\
S2 & 2-D/1-D & $0.70 / 0.69$ & - & $-11 / 2.10$ & $-6.08 /-5.98$ & - & - \\
S3 & 2-D/1-D & - & $2.08 / 0.14$ & $0.05 / 0.05$ \\
S4 & 2-D/1-D & - & $0.08 /-0.04$ & $0.04 / 0.01$ & $0.07 / 0.07$ & - & $-0.10 /-0.10$ \\
A1 & 2-D/1-D & - & $-4.21 /-4.15$ & - & - & $-0.17 /-0.17$ & - \\
A2 & 2-D/1-D & - & - & - & - & - \\
A3 & 2-D/1-D & - & - & - & - & - \\
A4 & 2-D/1-D & - & $0.09 /-0.11$ & - & - & - \\
\hline \hline
\end{tabular}




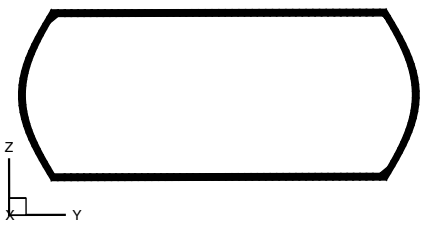

Mode 121

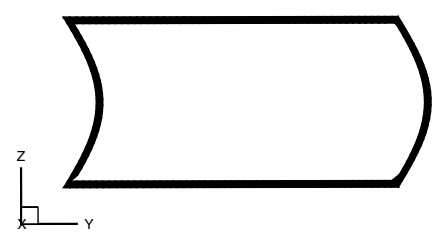

Mode 123

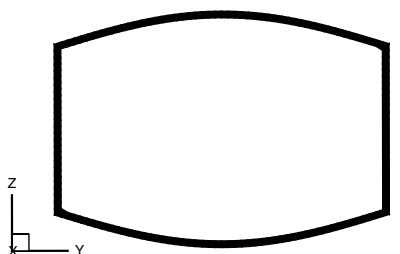

Mode 122

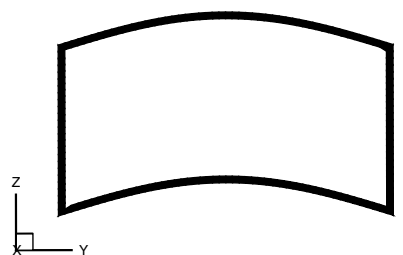

Mode 124

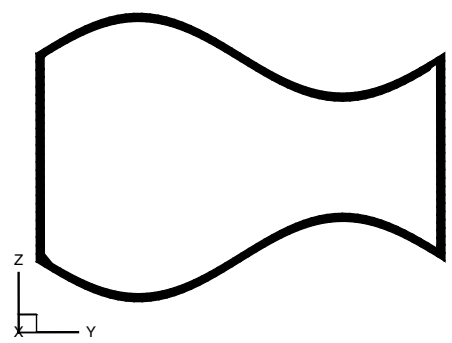

Mode 222

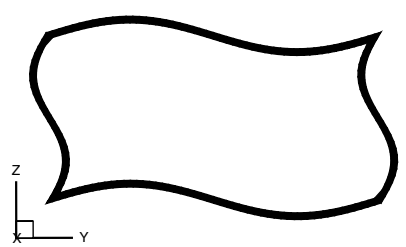

Mode 241

Fig. 6 Finite-section modes for the platelike deformations at box-beam walls.

shape function and the computed warping influence coefficients of Eq. (15), that is, $\Psi_{n}+w_{q_{n}}$. As with the classical deformation measures, the displacement field keeps the averaged value (the integral over the cross section) of the assumed shape functions.

With that information, the one-dimensional reduced model is now rebuilt and solved for the different configurations, including the finite-section modes shown in Fig. 6. The three-dimensional displacement field is constructed from both the cross-sectional and longitudinal results using Eq. (27). The resulting displacement field (magnified by a factor of 100) for several configurations defined in Table $\underline{3}$ is shown in Fig. $\underline{7}$, in which it is compared to the corresponding results from built-up shell finite-element models.

As it can be seen, the present approach captures both the longitudinal (long-scale) deformation as well as the local (smallscale) wall deformations for distributed loading, all within a onedimensional solution framework. Figure 8 includes a direct comparison of the deformed cross section at the center of the beam $(x=10 a)$ for cases A3 and A4. An excellent correlation can be observed. Regarding the selection of the finite-section modes, it is important to emphasize that the local minimization at the crosssectional level corrects the prescribed mode, and details in the original definition of the finite-section modes should not essentially affect the final solution.
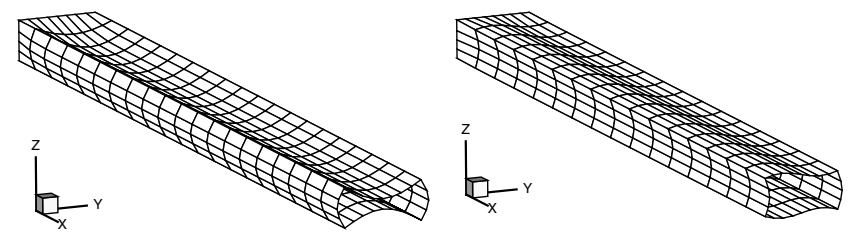

Case A3

Case A4
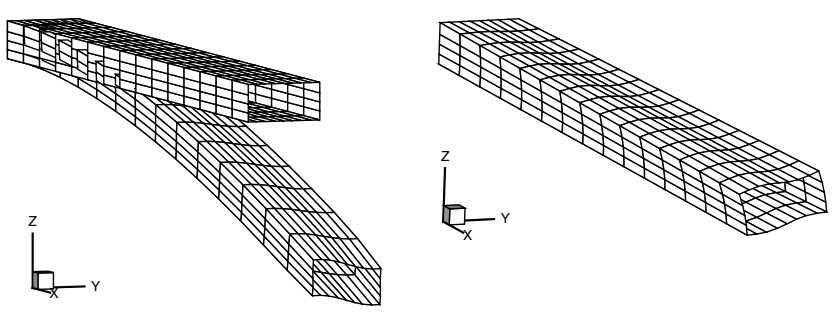

Case S3

Case S4

Fig. 7 Results for current one-dimensional model under active static loading (displacements magnified by 100).

\section{Linear Vibration Analysis of a Box Beam}

This example investigates the vibration characteristics of the aforementioned box beam (laminate $A$ in Table 3 ). Three different one-dimensional models are considered: 1) a generalized (classical) Euler-Bernoulli beam model, which includes only 4 elastic degrees of freedom (extension, twist, and bending in two directions) and 6 inertial degrees of freedom (three displacements and three rotations); 2) a generalized Timoshenko beam model, which adds the effect of transverse shear strains to the previous elastic description; and 3) the present approach, where the six additional cross-sectional deformations that were defined in Fig. $\underline{6}$ are added to the generalized Timoshenko model. Each finite-section mode adds two elastic states and one inertia state, defined by $q$ and $q^{\prime}$ in Eq. (16) and $\dot{q}$ in Eq. (17), respectively. Finally, results shown here correspond to $\sqrt{E_{11} / \rho a^{2}}=10^{4} s^{-1}$.

For lay up $A$, beam bending and twist responses are decoupled when the reference line is located at the area centroid. Therefore, the first vibration modes can be identified as VB, LB, T, or wall (W) modes. Table $\underline{5}$ includes the natural frequencies of the first 12 vibration modes, as obtained by the different structural models and the correlation index among the corresponding mode shapes, defined by the modal assurance criteria index.

These results show that significant improvements can be obtained with the additional one-dimensional degrees of freedom. At higher frequencies, local displacements appear on the beam walls, which are not captured by the two-dimensional warping influence coefficients of the Timoshenko DOF, that is, $w_{\gamma}$ and $w_{\kappa}$ in Eq. (15). Adding finite-section modes that explicitly account for the wall deformations provides a much better approximation to the actual vibration characteristics. A clear example of this is the third vertical bending mode, which is shown in Fig. 9 for the different models.

The lowest lateral bending modes involve displacements of the relatively stiffer lateral walls, and they show no significant local flexibility effects. Therefore, this is a situation in which the
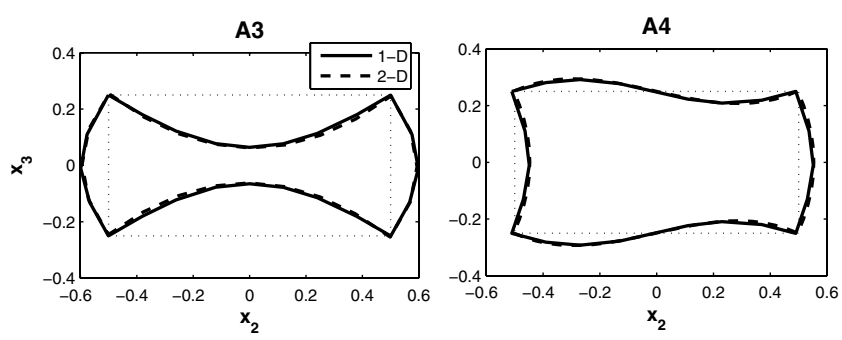

Fig. 8 Midspan cross-sectional deformation for actuation cases $A 3$ and A4 (displacements magnified by 100). 
Table 5 Natural frequencies (in $\mathrm{Hz}$ ) of laminate $A$.

\begin{tabular}{|c|c|c|c|c|c|c|c|c|c|c|c|}
\hline \multirow[b]{2}{*}{ Mode } & \multicolumn{2}{|c|}{ Shell } & \multicolumn{3}{|c|}{ One-dimensional Euler-Bernoulli } & \multicolumn{3}{|c|}{ (One-dimensional) Timoshenko } & \multicolumn{3}{|c|}{ One-dimensional with finite-section modes } \\
\hline & No. & Freq. & No. & Freq. & MAC & No. & Freq. & MAC & No. & Freq. & MAC \\
\hline VB1 & 1 & 3.34 & 1 & 3.34 & 0.998 & 1 & 3.32 & 0.999 & 1 & 3.33 & 0.998 \\
\hline $\mathrm{T} 1$ & 2 & 5.61 & 2 & 5.63 & 0.994 & 2 & 5.59 & 0.994 & 2 & 5.62 & 0.994 \\
\hline VB2 & 3 & 19.44 & 3 & 20.82 & 0.986 & 3 & 20.04 & 0.987 & 3 & 19.79 & 0.989 \\
\hline LB1 & 4 & 25.21 & 5 & 48.67 & 0.386 & 5 & 48.67 & 0.386 & 4 & 31.31 & 0.714 \\
\hline LB2 & 5 & 29.37 & 10 & 146.15 & 0.278 & 10 & 146.15 & 0.278 & 6 & 35.06 & 0.659 \\
\hline $\mathrm{T} 2$ & 6 & 33.29 & 4 & 34.68 & 0.961 & 4 & 33.36 & 0.965 & 5 & 33.35 & 0.961 \\
\hline W1 & 7 & 39.17 & - & - & - & - & - & - & 8 & 40.79 & 0.553 \\
\hline W2 & 8 & 39.92 & - & - & - & - & - & - & 9 & 41.37 & 0.494 \\
\hline VB3 & 9 & 39.99 & 6 & 57.91 & 0.872 & 6 & 53.24 & 0.878 & 7 & 40.13 & 0.912 \\
\hline W3 & 10 & 41.65 & - & - & - & - & - & - & 10 & 42.52 & 0.689 \\
\hline W4 & 11 & 42.95 & - & - & - & - & - & - & 11 & 44.21 & 0.845 \\
\hline W5 & 12 & 46.09 & - & - & - & - & 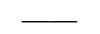 & - & 12 & 46.37 & 0.914 \\
\hline
\end{tabular}

Timoshenko BEST description of the one-dimensional deformation provides a very good approximation to the free vibration characteristics. This is no longer the case for the torsional modes, as shown in Table 5. Also, Fig. 10 shows the second torsional mode shape obtained by the different solutions. A substantial difference can be observed between the solutions given by the different onedimensional formulations. This occurs because the description of the deformation used in beam models based on the classical BEST DOF does not capture the relatively large warping deformations associated with the torsional dynamics of a thin-walled beam. A much better approximation was found by adding additional elastic degrees of freedom through finite-section modes, as shown in Fig. 10. However, despite the improvement, there is still a $20 \%$ error in the estimation of the natural frequencies with respect to the shell model. Two main reasons for this error are as follows. First, finite-section modes are by definition approximations to the actual elastic field, and therefore carry some error with them. Adding additional elastic states may improve the results. And second, the present theory does not include Vlasov's warping constraint in the torsional curvature, as described for instance by Volovoi and Hodges [17], which is believed to be responsible for the errors in the torsional modes.

Finally, one can also identify a number of modes at low frequencies that essentially involve the motion of the walls. These modes are not captured by the Timoshenko DOF and are referred to here as "wall" modes. They were included in Table 5 , and their mode shapes are shown in Fig. 11, as obtained by the one- and two- dimensional models. In general, an acceptable correlation can be observed among both solutions, with a higher one in the estimation of the vibration frequencies (with errors below 4\%) than on the modal shapes (with MAC numbers as low as 0.5). This has been done with only a few additional one-dimensional elastic degrees of freedom (i. e., the finite-section modes in Fig. 6). As with the torsional modes, the one-dimensional model fails to capture accurately the constrained warping at the clamped end. The effect of higher-order spatial derivatives of the finite-section amplitudes in reducing this error should be investigated in further extensions of this theory (this formulation was based on $q$ and $q^{\prime}$ contributions). In addition to this, further refinement of these results is, in principle, always possible by expanding the number of finite-section modes in the approximation to the warping field.

As a final remark, it should be noted that all previous lowfrequency modes of the box beam could be obtained with only three of the finite-section modes introduced in Fig. 6, that is, modes 122, 124, and 241. Furthermore, mode 241 was seen to be linked to the twist curvature, and they could in principle be combined into a single elastic degree of freedom in the one-dimensional model. This was not done here to show the generality of the formulation.

\section{Conclusions}

The conventional BEST beam model has been expanded to allow arbitrary deformations of the finite-size cross sections. To achieve

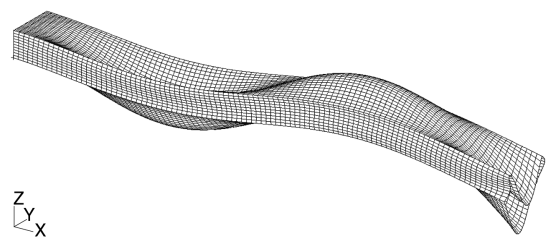

Shell model. Mode 9

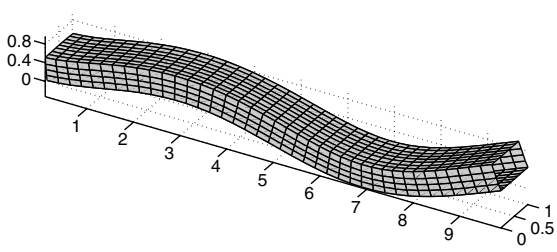

Timoshenko 1-D. Mode 6

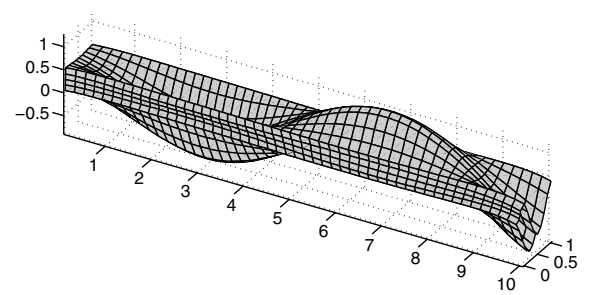

1-D with finite section. Mode 7

Fig. 9 Third vertical (flapwise) bending mode shape of laminate $A$.

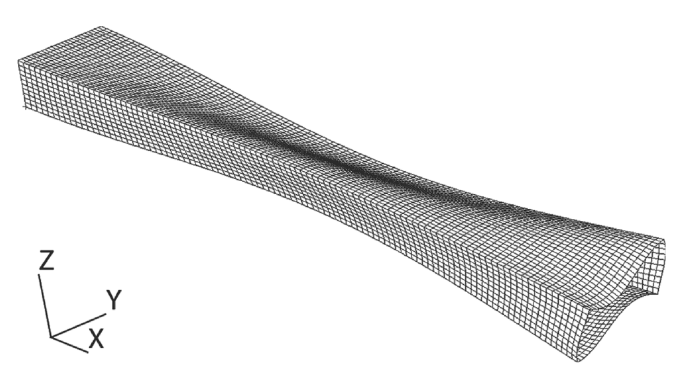

Shell model. Mode 5

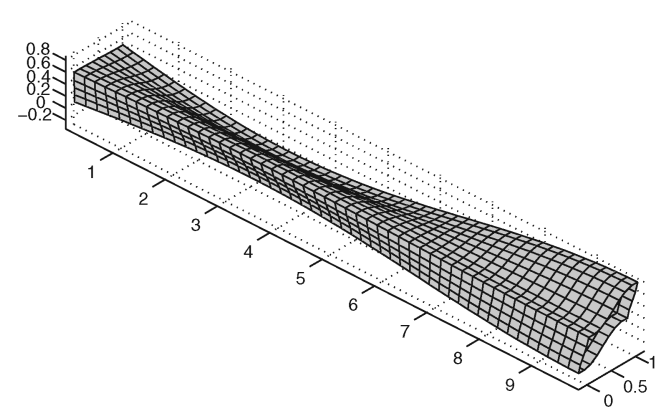

1-D with finite section. Mode 5

Fig. 10 Second torsional mode shape of laminate $A$. 


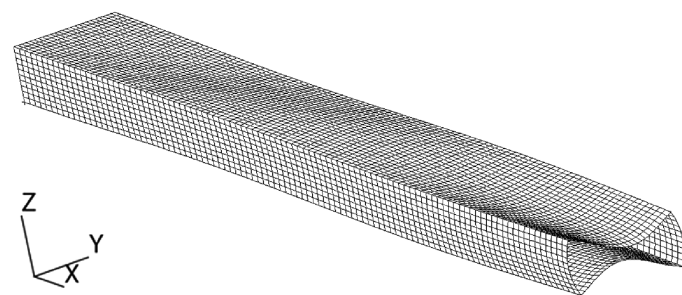

Shell model. Mode 7

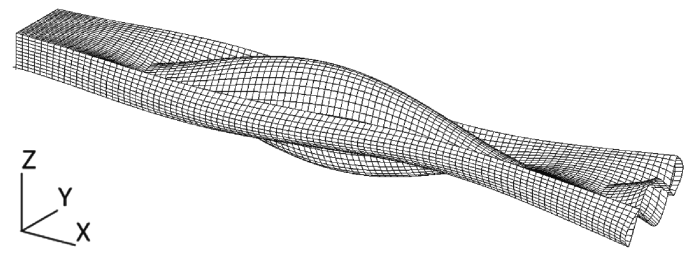

Shell model. Mode 8

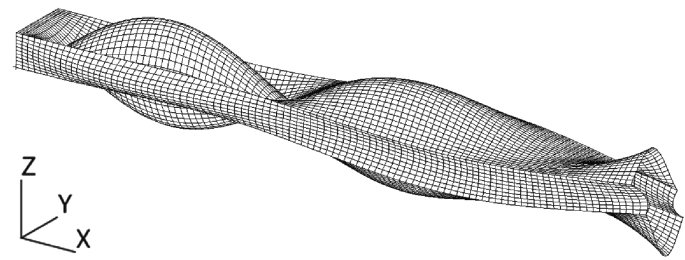

Shell model. Mode 10

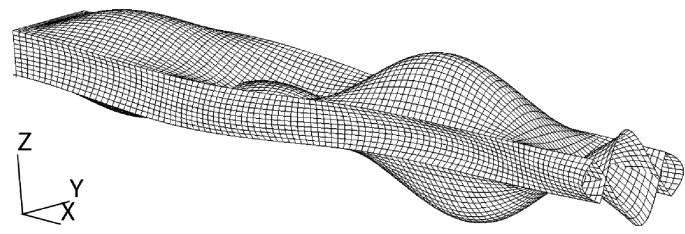

Shell model. Mode 11

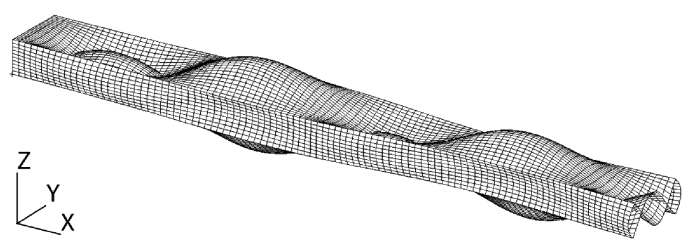

Shell model. Mode 12

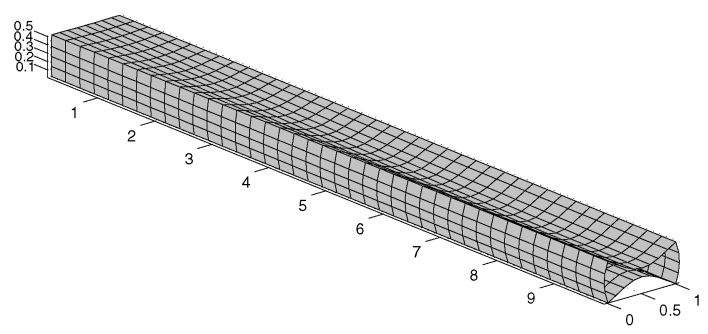

1-D with finite section modes. Mode 8

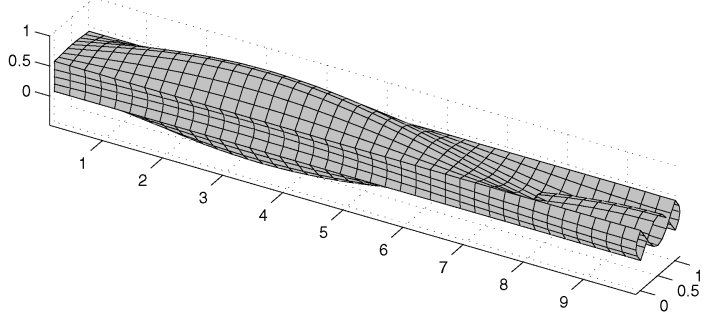

1-D with finite section modes. Mode 9

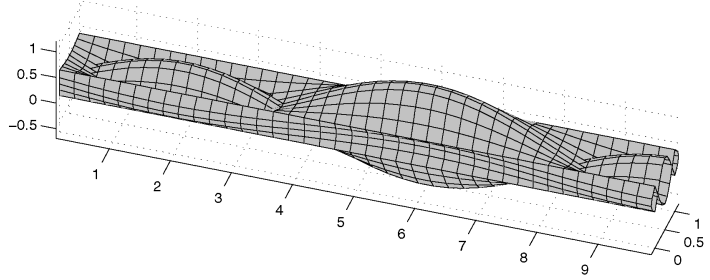

1-D with finite section modes. Mode 10

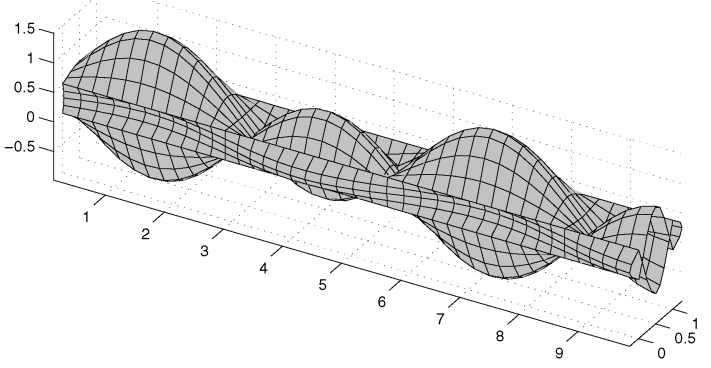

1-D with finite section modes. Mode 11

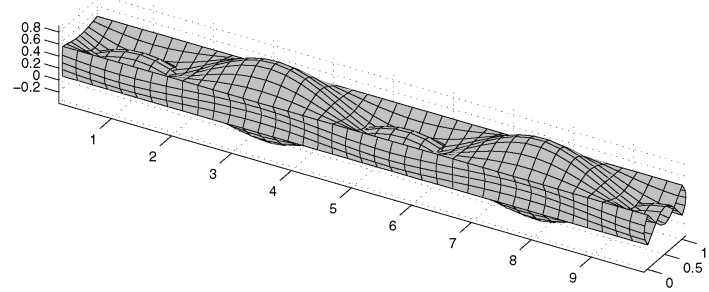

1-D with finite section modes. Mode 12

Fig. 11 Wall mode shapes of laminate $A$.

this, a set of additional one-dimensional degrees of freedom has been introduced in the model by means of approximating functions (finitesection modes) to the warping displacement field. A spatial homogenization process has determined the reduced one-dimensional description of the dynamics of a general anisotropic slender structure that, in addition to the conventional sectional forces and moments, also includes the force conjugates to those finite-section modes. This results in a geometrically nonlinear theory of heterogeneous anisotropic beams with deformable cross sections of arbitrary shape. Finite-element solutions at both the cross-sectional and longitudinal problems define a robust numerical solution process for the analysis of complex configurations. For composite beams, this refinement in the kinematical description may provide a significant improvement in accuracy with very little modeling or computational cost.
Numerical results using this approach have shown the power of this approach. They compared very well with finite-element shell models for isotropic thin strip and composite box-beam cases presented here. Only a few finite-section modes, which are defined numerically (in the examples, harmonic shapes for the box beam and parabolic deformation for the thin strip), are needed to obtain a rather complete description of the three-dimensional solid deformations, both under distributed loads and in the low-frequency linear vibration response. In the dynamic response, the additional finitesection modes allow not only the capture of vibration modes that are invisible by conventional beam models (wall modes), but also improved the results corresponding to the bending and torsion modes. This latter improvement can be explained because beam models based on the BEST description are obtained by homogenization in the spatial variables of the three-dimensional 
solid mechanics equations, rather than in both space and time. This quasi-static approach to evaluating the internal energy neglects the inertia of the cross-sectional warping and is a source of error in the estimation of the vibration characteristics of the actual threedimensional solid. Adding finite-section modes allows the circumvention of this limitation, as the contribution of the warping deformation is included both to the strain and the kinetic energy of the beam. However, this is still done within the scope of a purely spatial cross-sectional reduction process, to ease both the theoretical formulation and the numerical implementation.

\section{Acknowledgments}

This work is supported by the Georgia Institute of Technology/ University of Michigan/Washington University Vertical Lift Rotorcraft Center of Excellence (VLRCOE) funded by the U.S. Army. Michael Rutkowski is the technical monitor.

\section{References}

[1] Volovoi, V. V., Hodges, D. H., Cesnik, C. E. S., and Popescu, B., "Assessment of Beam Modeling Methods for Rotor Blade," Mathematical and Computer Modelling, Vol. 33, Nos. 10-11, 2001, pp. 1099-1112. doi:10.1016/S0895-7177(00)00302-2

[2] Jung, S. N., Nagaraj, V. T., and Chopra, I., "Assessment of Composite Rotor Blade Modeling Techniques," Journal of the American Helicopter Society, Vol. 44, No. 3, 1999, pp. 188-205.

[3] Berdichevsky, V. L., "On the Energy of an Elastic Rod," Journal of Applied Mathematics and Mechanics, Vol. 45, No. 4, 1981, pp. 518529.

[4] Cesnik, C. E. S., and Hodges, D. H., "VABS: A New Concept for Composite Rotor Blade Cross-Sectional Modeling," Journal of the American Helicopter Society, Vol. 42, No. 1, 1997, pp. 27-38.

[5] Le, K. C., Vibrations of Shells and Rods, Springer-Verlag, Berlin, 1999.

[6] Buannic, N., and Cartraud, P., "Higher-Order Effective Modeling of Periodic Heterogeneous Beams 1: Asymptotic Expansion Method," International Journal of Solids and Structures, Vol. 38, Nos. 40-41, 2001, pp. 7139-7161. doi:10.1016/S0020-7683(00)00422-4

[7] Fan, H., and Widera, G. E. O., "Refined Engineering Beam Theory Based on the Asymptotic Expansion Approach," AIAA Journal, Vol. 29, No. 3, 1991, pp. 444-449.

[8] Parker, D. F., "An Asymptotic Analysis of Large Deflections and Rotations of Elastic Rods," International Journal of Solids and Structures, Vol. 15, No. 5, 1979, pp. 361-377. doi:10.1016/0020-7683(79)90059-3

[9] Love, A. E. H., Mathematical Theory of Elasticity, Dover, New York, 1944.

[10] Gruttmann, F., and Wagner, W., "Shear Correction Factors in Timoshenko's Beam Theory for Arbitrary Shaped Cross-Sections," Computational Mechanics, Vol. 27, No. 3, 2001, pp. 199-207. doi:10.1007/s004660100239

[11] Friedman, Z., and Kosmatka, J. B., "Torsion and Flexure of a Prismatic Isotropic Beam Using the Boundary Element Method," Computers and Structures, Vol. 74, No. 4, 2000, pp. 479-494. doi:10.1016/S0045-7949(99)00045-0

[12] Kosmatka, J. B., "Extension-Bend-Twist Coupling Behavior of NonHomogeneous Anisotropic Beams with Initial Twist," AIAA Journal,
Vol. 30, No. 2, 1992, pp. 519-527.

[13] Giavotto, V., Borri, M., Mantegazza, P., Giringhelli, G., Carmaschi, V., Maffioli, G. C., and Mussi, F., "Anisotropic Beam Theory and Applications," Computers and Structures, Vol. 16, Nos. 1-4, 1983, pp. 403-413.

doi:10.1016/0045-7949(83)90179-7

[14] Cesnik, C. E. S., Sutyrin, V. G., and Hodges, D. H., "Refined Theory of Composite Beams: The Role of Short-Wavelength Extrapolation," International Journal of Solids and Structures, Vol. 33, No. 10, 1996, pp. 1387-1408. doi:10.1016/0020-7683(95)00109-3

[15] Palacios, R., Cesnik, C. E. S., "Cross-Sectional Analysis of Nonhomogeneous Anisotropic Active Slender Structures," AIAA Journal, Vol. 43, No. 12, 2005, pp. 2624-2638.

[16] Palacios, R., and Cesnik, C. E. S., "On the One-Dimensional Modeling of Camber Bending Deformations in Active Anisotropic Slender Structures," International Journal of Solids and Structures (to be published).10.1016/j.ijsolstr.2007.11.011

[17] Volovoi, V. V., and Hodges, D. H., "Theory of Anisotropic ThinWalled Beams," Journal of Applied Mechanics, Vol. 67, No. 3, 2000, pp. 453-459. doi:10.1115/1.1312806

[18] Reissner, E., "On One-Dimensional Large-Displacement Finite-Strain Beam Theory," Studies in Applied Mathematics, Vol. 52, No. 2, 1973, pp. 87-95.

[19] Simo, J. C., "Finite Strain Beam Formulation: The Three-Dimensional Dynamic Problem-Part 1," Computer Methods in Applied Mechanics and Engineering, Vol. 49, No. 1, 1985, pp. 55-70. doi:10.1016/0045-7825(85)90050-7

[20] Hodges, D. H., "A Mixed Variational Formulation Based on Exact Intrinsic Equations for Dynamics of Moving Beams," International Journal of Solids and Structures, Vol. 26, No. 11, 1990, pp. 1253-1273. doi:10.1016/0020-7683(90)90060-9

[21] Hodges, D. H., Shang, X., and Cesnik, C. E. S., "Finite Element Solution of Nonlinear Intrinsic Equations for Curved Composite Beams," Journal of the American Helicopter Society, Vol. 41, No. 4, 1996, pp. 313-321.

[22] Renaud, T., O’Brien, D. M., Smith, M. J., and Potsdam, M., "Evaluation of Isolated Fuselage and Rotor-Fuselage Interaction Using CFD," Proceedings of the 60th Annual Forum of the American Helicopter Society, American Helicopter Society, Alexandria, VA, June 2004, pp. 995-1009.

[23] Peters, D. A., Hsieh, M. A., and Torrero, A., "A State-Space Airloads Theory for Flexible Airfoils," Proceedings of the 62nd Annual Forum of the American Helicopter Society, American Helicopter Society, Alexandria, VA, May 2006, pp. 1806-1823.

[24] Hodges, D. H., Nonlinear Composite Beam Theory, Progress in Aeronautics and Astronautics, Vol. 213, AIAA, Reston, VA, 2005.

[25] Danielson, D. A., and Hodges, D. H., "Nonlinear Beam Kinematics by Decomposition of the Rotation Tensor," Journal of Applied Mechanics, Vol. 54, No. 2, 1987, pp. 258-262.

[26] Zienkiewicz, O. G., and Taylor, R. L., The Finite-Element Method, 5th ed., Butterworth-Heinemann, Oxford, U.K., 2000.

[27] Lehoucq, R. B., Sorensen, D. C., and Yang, C., ARPACK Users' Guide: Solution of Large-Scale Eigenvalue Problems with Implicitly Restarted Arnoldi Methods, SIAM, Philadelphia, USA, 1998.

[28] Chopra, I., "Review of State-of-Art of Smart Structures and Integrated Systems," AIAA Journal, Vol. 40, No. 11, 2002, pp. 2145-2187.

M. Hyer

Associate Editor 